\title{
Evaluation of Expert Systems Techniques for Classifying Different Stages of Coffee Rust Infection in Hyperspectral Images
}

\author{
Wilson Castro $^{1}$, Jimy Oblitas $^{2}$, Jorge Maicelo ${ }^{3}$, Himer Avila-George ${ }^{4}$ \\ ${ }^{1}$ Facultad de Ingeniería, Universidad Privada del Norte, \\ Cajamarca, Cajamarca 06002, Perú. \\ E-mail:wilson.castro@upn.edu.pe \\ ${ }^{2}$ Centro de Investigaciones e Innovaciones de la Agroindustria Peruana (CIIAP), \\ Amazonas, 1061, Perú. \\ E-mail: j_oblitas@hotmail.com \\ ${ }^{3}$ Facultad de Ingeniería Zootecnista, Agronegocios y Biotecnología, Universidad Nacional Toribio Rodríguez \\ de Mendoza de Amazonas, Chachapoyas, Chachapoyas 01001, Perú. \\ E-mail: jmaicelo@indes-ces.edu.pe \\ ${ }^{4}$ Unidad de Transferencia Tecnológica Tepic, CONACYT-CICESE, \\ Tepic, Nayarit 63173, México. \\ E-mail: himerag@cicese.mx
}

Received 30 November 2016

Accepted 17 September 2017

\begin{abstract}
In this work, the use of expert systems and hyperspectral imaging in the determination of coffee rust infection was evaluated. Three classifiers were trained using spectral profiles from different stages of infection, and the classifier based on a support vector machine provided the best performance. When this classifier was compared to visual analysis, statistically significant differences were observed, and the highest sensitivity of the selected classifier was found at early stages of infection.
\end{abstract}

Keywords: Expert systems, Hyperspectral images, Coffee rust infection, Spectral profiles.

\section{Introduction}

It is well known that both physiological and infectious plant diseases can have critical impacts on agricultural production and economic losses worldwide. ${ }^{1,2,3}$ Common sources of infection include insects and microorganisms such as bacteria, fungi, and viruses. Fungi are the most diverse group of plant pathogens; there are more than 20,000 species of fungi that can cause diseases in crops and plants, and these fungi are responsible for
$70-80 \%$ of plant diseases. ${ }^{4}$

Crop loss due to plant diseases may cause food insecurity and famines ${ }^{1}$; consequently, detecting plant diseases and their pathogens is an operation of primary importance to agricultural field management ${ }^{5,6}$ and an essential research topic in agriculture. ${ }^{3}$ Given this scenario, there is a need for research to focus on the rapid design of tools for the early detection of symptoms of a particular disease. ${ }^{3,2}$ These requirements and the recent developments in agricultural technology have led to a de- 
mand for a new era of automated, non-destructive methods for detecting plant diseases. ${ }^{1,2,7}$

The traditional methods for detecting fungal pathogens in plants involve interpreting the visual symptoms of the disease or identifying the pathogens that cause the disease. To understand the visual symptoms of a particular disease, persons skilled in the disease are used. ${ }^{3,4,5,6}$ To identify the pathogens that cause a particular disease, morphological, microbiological and biochemical techniques may be used. ${ }^{4}$

However, visual inspection methods are subjective, occasionally inconsistent and generally slow, and given their high cost, such methods are often prohibitive on large farms. Meanwhile, laboratory analyses, such as molecular, immunological or pathogen culturing-based approaches, are often time consuming and destructive. ${ }^{6,8}$

In this context, it is compelling to develop nondestructive, automated methods that are capable of identifying diseases in a rapid and reliable way and that are capable of detecting early infections; ${ }^{6,9}$ thus, new techniques such as digital images that can extract information of symptoms in the visible and near-infrared (NIR) bands are receiving attention. ${ }^{6}$ In these ranges, according to Refs. 10-12, the spectrum of plant leaves changes in both the visible and the near-infrared regions due to physiological stress. Furthermore, metabolic disturbances were found to affect the ability of chlorophyll to absorb visible light, and with the deterioration of the metabolism, the visible reflectance increases. It was also reported that the reflectance of a leaf in the NIR region decreases in advanced disease infestation as actual breakdown of the leaf occurs. Thus, it is not surprising that methods for automatic plant disease diagnosis based on visible range digital images have received special attention. ${ }^{13,14}$

Using the spectrum variations according to the health conditions of the plant and the assumption that objects with the same spectral characteristics should belong to the same class and be assigned to one characteristic appearance in the final classification map requires a procedure that assigns each pixel to one of other predefined classes. ${ }^{12}$ Accordingly, several studies have shown the capabilities of red green blue (RGB), multispectral images (MSIs) and hyperspectral images (HSIs) for detecting fungal diseases in crops, as shown in table ${ }_{n} e w$.

Hyperspectral imaging is the most powerful technique since it can capture both spectral and imaging information for the same object covering hundreds of wavebands, which allow determining the physical, morphological and chemical characteristics and molecular information of materials. HSIs are formed by a high number of intensity images, commonly named channels, in a three-dimensional array called a hypercube, ${ }^{26}$ which includes some regions that are not detectable by the human eye, such as ultraviolet, near infrared, and infrared. This characteristic is of considerable interest for its application in precision agriculture ${ }^{2}$ disease evaluation. ${ }^{4}$ However, this technique requires the use of special methods, such as those based on machine learning techniques such as artificial neural networks (ANNs), decision tree (DT), K-means (KM), Knearest neighbor (KNN), and support vector machine (SVM), for data analysis. These techniques have been applied in agricultural research and shown potential for automatic classification methods in site-specific weed detection. ${ }^{7}$

Yellow coffee rust, which is caused by the fungus Hemileia vastatrix $B \& B$, is one of the most economically important diseases affecting coffee $^{27,28,29}$; it reduces the photosynthetic activity and causes defoliation of the plant. ${ }^{30}$ This disease causes worldwide economic losses of approximately $\$ 1$ billion US dollars due to high mitigation costs and the decrease in productivity of up to fifty percent. ${ }^{28,31}$ Thus, to prepare mitigation actions, it is necessary to evaluate rust damage during the early stages of infection. ${ }^{32}$ Therefore, in the diagnosis of plant fungal infections, it is essential to perform a correct disease detection process, ${ }^{4}$ particularly using non-destructive and real-time techniques. ${ }^{1}$

Consequently, the main objective of this work was to evaluate the use of expert systems techniques and hyperspectral imaging to classify different stages of coffee rust infection in coffee (Coffea Typica) leaves. 
Table 1. Evaluation of fungal diseases using image analysis coupled to spectroscopic technology.

\begin{tabular}{|c|c|c|c|c|c|c|}
\hline State & Disease/Pest & Technique & Model & $\begin{array}{r}\text { Wavelength } \\
(\mathrm{nm})\end{array}$ & $\begin{array}{r}\text { Results } \\
(\text { accuracy \%) }\end{array}$ & Source \\
\hline Eggplant leaves & Botrytis cinerea & HSI & ANN & $400-900$ & 70.5 & Ref. 15 \\
\hline Rice leaves & Helminthosporium oryzae & HSI & RA & $400-2,350$ & 96 & Ref. 5 \\
\hline Citrus & Penicillium digitatum & HSI & NLBDA & $400-1,800$ & 97 & Ref. 16 \\
\hline Maize kernel & Fusarium & HSI & PLS-DA & $1,000-2,498$ & 85 & Ref. 17 \\
\hline Rice panicles & Phoma sorghina & HSI & ANN & $350-2,500$ & $>91$ & Ref. 18 \\
\hline Leafs & Sclerotinia & RGB & ANN & - & $>96$ & Ref. 3 \\
\hline Cotton & Phymatotrichum omnivorum & HSI - MSI & ISODATA & $400-1,000$ & $>96$ & Ref. 19 \\
\hline Wheat leaves & Fusarium & HSI & SAM & $400-1,000$ & 87 & Ref. 20 \\
\hline Wheat leaves & Blumeria graminis & HSI & MLR / PLSR & $450-950$ & $>90$ & Ref. 21 \\
\hline Wheat & Fusarium & HSI & PLS-DA & $1,000-1,700$ & $>91$ & Ref. 8 \\
\hline Wheat leaves & Puccinia striiformis & Spectroradiometer & PLSR & $350-2,500$ & $>89$ & Ref. 22 \\
\hline Sugar beet & Cercospora beticola & RGB & SVM & - & $>93$ & Ref. 23 \\
\hline Wheat kernels & Fusarium & MSI & MLR & $360-950$ & - & Ref. 24 \\
\hline Tomato leaves & Alternaria solani - Phytophthora infestans & HSI & ELM & $380-1,023$ & $>97$ & Ref. 25 \\
\hline Strawberry leaves & Colletotrichum gloeosporioides & HSI & $\mathrm{SAM} / \mathrm{SDA} / \mathrm{CM}$ & $460-930$ & $>80$ & Ref. 1 \\
\hline
\end{tabular}

RA = Ratio Analysis; NLBDA = Non-linear Bayesian Discriminant Analysis; PLS-DA = Partial Least Squares and Discriminant Analysis; ISODATA = Iterative Self-Organizing Data Analysis; LDA = Linear Discriminant Analysis; MLR = Multiple Linear Regression; PLSR = Partial Least Squares Regression; ELM = Extreme Learning Machine; SAM = Spectral Angle Mapper;

SDA $=$ Stepwise Discriminant Analysis; $\mathrm{CM}=$ Correlation Measure .

\section{Materials and Methodology}

\subsection{System for acquiring hyperspectral images}

The system for acquiring hyperspectral images was obtained from RESONON Inc., model PIKA XC (see fig1).

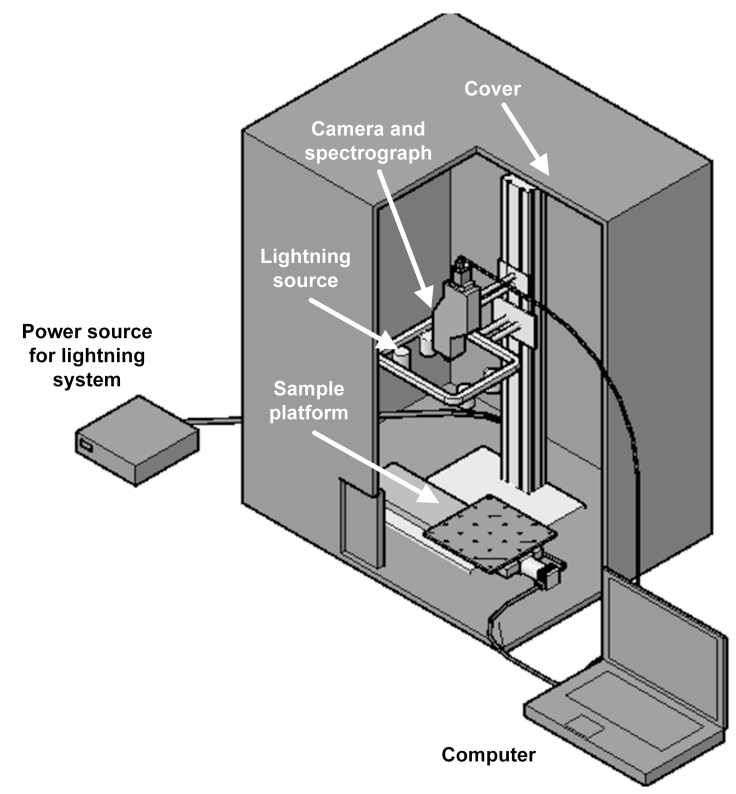

Fig. 1. Hyperspectral imaging system.
The elements that compose the system for hyperspectral image acquisition are as follows:

- Camera and spectrograph. Filtering of the wavelengths at which to acquire the intensity images.

- Cover. To avoid the influence of exterior lighting.

- Computer. To manage the image acquisition software.

- Power source. Provides energy for light source.

- Light source. Composed of four halogen lights arranged at variable and adjustable height.

- Sample platform. For horizontal scrolling during speed-controlled scanning.

This system operates in a reflectance mode with scanning line by line (pushbroom) in the range of $400-1,000 \mathrm{~nm}$, obtaining intensity images at intervals of $8 \mathrm{~nm}$ (76 images per scene). These images are arranged in a three-dimensional matrix, called a hypercube, ${ }^{26}$ whose extension is ".bil" (band interleaved by line) and requires a header file whose extension is ".hdr".

\subsection{Samples}

The sample was composed of 140 coffee leaves (var. Typica); these leaves were collected from a coffee plantation located in the district of San Nicolas, 
province of Rodríguez de Mendoza in the Amazons region [-6.40144, -77.50135 U.T.M.]. The sample contains healthy leaves and diseased leaves at different levels of infection with rust. table1 shows the sample, and in fig2, the progression of rust infection in coffee leaves is shown as a reference.

Table 2. Distribution of the coffee leaves that composed the sample.

\begin{tabular}{llr}
\hline \multirow{2}{*}{ State } & \multicolumn{2}{c}{ Number of leaves for classifier } \\
\cline { 2 - 3 } & Generation & Application \\
\hline Healthy & 5 & 12 \\
Sick & 15 & 108 \\
\hline Total & 20 & 120 \\
\hline
\end{tabular}

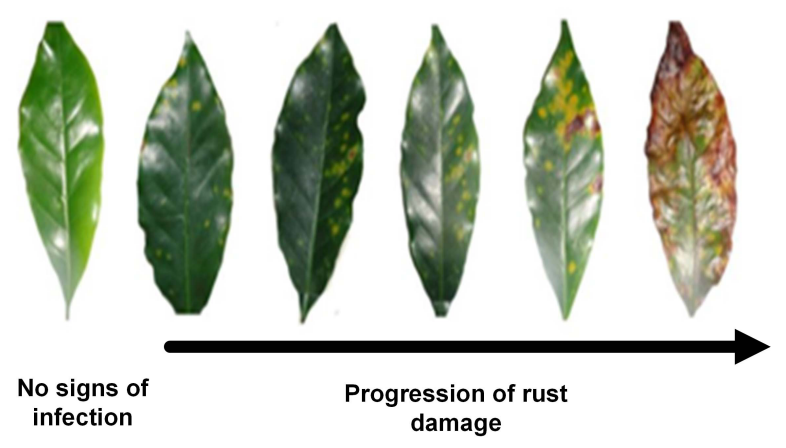

Fig. 2. Progression of rust infection in coffee leaves.

\subsection{Methodology}

The methodology proposed in this research is based on the methods proposed by Refs. 1, 32, and 33 . This methodology focuses on the generation, validation, and comparison of classifiers, and it is described in fig3.

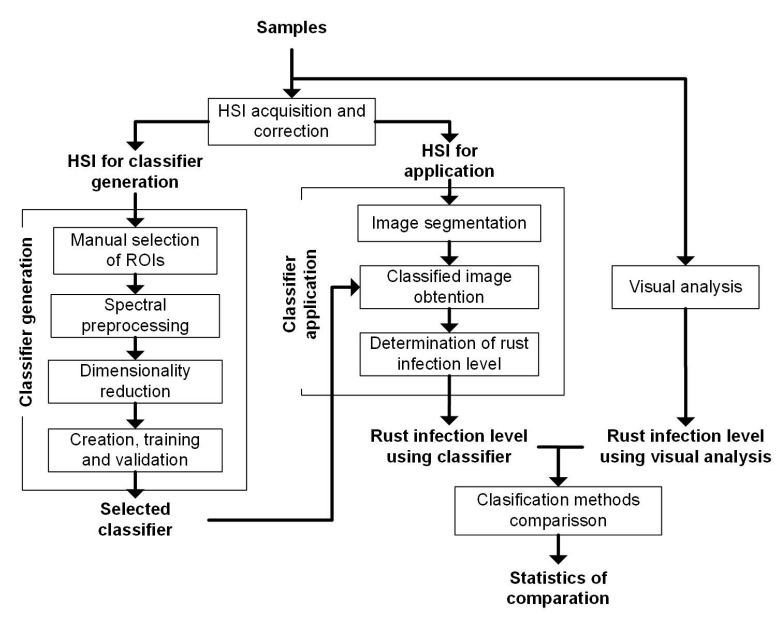

Fig. 3. Experimental methodology.

In the following subsections, the stages and steps used are explained in detail.

\subsubsection{HSI acquisition and correction}

First, the system parameters for HSI acquisition were set as follows: speed of sample platform $=$ $0.5 \mathrm{~cm} / \mathrm{s}$, distance to sample surface $=28.3 \mathrm{~cm}$, and diaphragm aperture $=6.4 \mathrm{~mm}$. Then, using the software SpetrononPro 2.62, the hyperspectral images of each leaf were obtained.

Subsequently, the HSIs were spatially corrected according to the methods proposed by Refs. 34, 35, and 36. For this purpose, eq01 was used, and the values of white reference image intensity $(W)$ were acquired from a Teflon pattern (reflectance value of $\sim 99.9 \%$ ), and the black reference $(D)$ was acquired by blocking the lens (reflectance of $\sim 0.0 \%$ ).

$$
\forall P_{i j} \in I(x, y, \lambda): P_{i j}^{\prime}=\frac{P_{i j}-D_{i j}}{W_{i j}-D_{i j}} .
$$

Here, $P_{i j}$ is the value of the pixel at position $(i j)$ in the raw image $I(x, y, \lambda)$ at all wavelengths $(\lambda) . P_{i j}^{\prime}$ is the pixel value in the corrected image $I^{\prime}(x, y, \lambda)$, $D_{i j}$ is the value of the pixel in the black reference image, and $W_{i j}$ is the value of the pixel in the white reference.

\subsubsection{Classifier generation}

At this stage, three classifiers were generated using spectral information from the different levels of dis- 
ease progression; the steps followed are detailed below.

Manual selection of regions of interest (ROIs). In each leaf, by using its image in RGB format, ROIs were selected based on the visual appearance. For this purpose, five levels of rust infection and its visual signs were pre-established; see fig ${ }_{t}$ ableand fig 4 .

Table 3. Visual scale for rust infection level in coffee leaves.

\begin{tabular}{|c|c|c|}
\hline Level & Stage & Visual appearance of tissue \\
\hline 0 & Healthy & Characteristic color \\
\hline 1 & Initial & $\begin{array}{l}\text { Small round chlorotic spot, diffuse } \\
\text { edges }\end{array}$ \\
\hline 2 & Intermediate & Chlorotic border spot defined \\
\hline 3 & Advanced & $\begin{array}{l}\text { Chlorotic spot with initial signs of } \\
\text { necrosis, brown coloration }\end{array}$ \\
\hline 4 & Necrotic & Brown fabrics \\
\hline
\end{tabular}

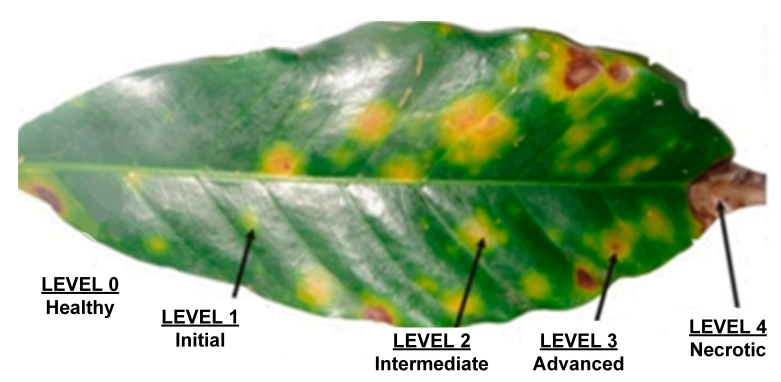

Fig. 4. Example of visual signs for rust infection in coffee leaves.

Spectral profiles of each level of damage were obtained using the ROI selection method proposed by Ref. 20, which is summarized in fig5, and a graphical user interface (GUI) developed and implemented for this purpose in Matlab 2015a. This GUI used the position of each pixel in manually selected ROIs correlating with its levels of rust infection.

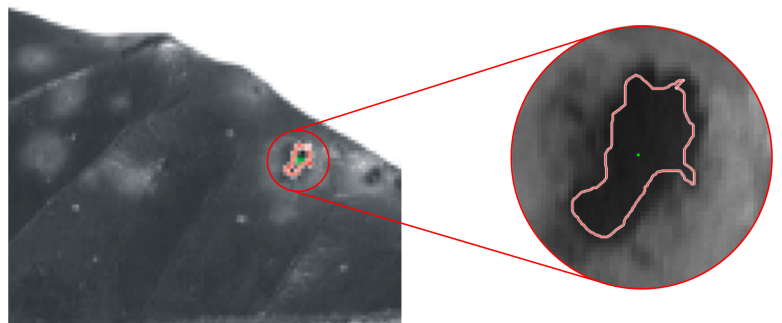

a)

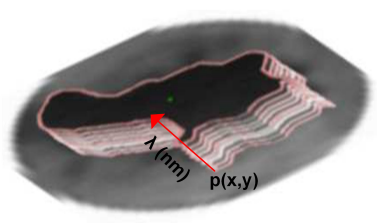

c)

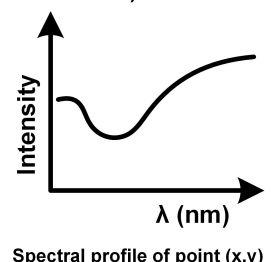

d)
Fig. 5. (a) Section containing ROI; (b) Increase of section; (c) Manual drawing of ROI; (d) Obtaining spectral profiles.

This method is compared with the works of Refs. 37, 38, and 39 based on the selection of rectangular ROIs or with the methods used by Refs. 40 or 41 based on a difference in contrast, allowing different stages in tissues to be followed and improving information for the training of classifiers.

However, because this method could generate errors through the border effect or irregular distribution of damage levels in the selected tissue, it is necessary to pre-process the spectral profiles to reduce noise and remove anomalous profiles.

Spectral preprocessing. In most cases, the extracted spectral data contain some noise and variability, and addressing this variability requires the use of spectral enhancement, such as spectral filtering, smoothing, normalization, mean centering, and auto scaling. ${ }^{12}$

For this research, the spectral profiles were first smoothed using a second-degree Savitzky-Golay filter; see eq.2.

$$
Y_{j}^{o}=\frac{\sum_{i=-m}^{m} C_{i}, y_{j+i}}{N} .
$$

Here, $Y$ is the original profile, $Y_{o}$ is the filtered profile, $C$ is the coefficient for the $i^{t h}$ term of the profile, and $N$ is the number of convolution integers. 
Subsequently, anomalous spectral profiles were removed using a script that evaluates intensity values at each wavelength and deletes those whose intensity deviates from the median by more than one standard deviation. This script uses the following steps:

(i) Select wavelength $\left(\lambda_{i}\right)$.

(ii) Calculate the median $(\widehat{I})$ and standard deviation $\left(\sigma_{I}\right)$ of the intensity value at $\left(\lambda_{i}\right)$.

(iii) Select a profile $(p)$.

(iv) If $I_{p}<\widehat{I}-\sigma_{I}$ or $I_{p}>\widehat{I}+\sigma_{I}$, delete profile $(p)$; otherwise, keep profile $(p)$.

(v) Return to point (item iii) until finishing the profiles.

(vi) Return to point a until the wavelengths are over.

Dimensionality reduction. In many situations, dimensionality reduction can improve model performance and model characteristics by identifying and removing useless, noisy and redundant variables. ${ }^{42}$ Therefore, in this research, the possibility of dimensionality reduction was evaluated using the Bartlett test and Kaiser-Meyer-Olkin (KMO) test. Subsequently, following the reports of Refs. 43, 44, 45, and 46 , the dimensionality of the input data was reduced using principal component analysis (PCA).

PCA reduces the dimensions of the data, transforming the initial dimensions into new uncorrelated dimensions called main components through a linear combination of the initial dimensions, and these are obtained in order of importance according to their capacity to represent the greater variability of the data. ${ }^{43}$ This step was performed using the $p c a$ function of Matlab 2015a.

Creation, training and validation of classifiers. Using the Matlab 2015a classification learner toolbox, three different classifiers were created and trained: support vector machine (SVM), decision tree (DT), and K-nearest neighbor (KNN).

From each classifier, confusion matrices and the principal statistics values were obtained to select the classifier with the best performance.

\subsubsection{Classifier application}

At this stage, the potential of the expert system previously selected to determine the progress of leaf rust infection was evaluated in comparison to the visual analysis performed by experts.

The first step was the segmentation of the images. In this step, the pixels of the image containing coffee leaf information were selected using the contrast between the sample and background; see eq. 7 .

$$
g_{(x, y)}=\left\{\begin{array}{ll}
1 & p_{(x, y)} \geqslant T \\
0 & p_{(x, y)} \leqslant T
\end{array} .\right.
$$

Here, $g$ is the binary image, $p_{(x, y)}$ represents the intensity of the image in gray scale, and $T$ is the threshold. In this step, intensity images were selected at $562 \mathrm{~nm}$ because greater contrast between sample and background exists at this wavelength; see fig6.

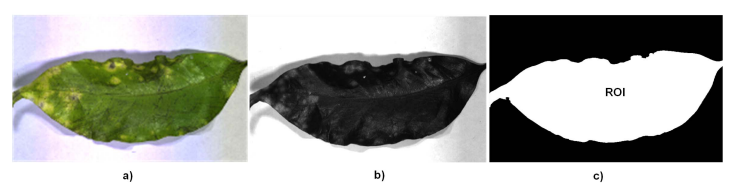

Fig. 6. Leaf image: a) RGB, b) $562 \mathrm{~nm}$, and c) segmented.

To obtain classified images, according to the level of damage in the coffee leaves, a pixel by pixel analysis is conducted to determine the affected area over the entire sample. For this purpose, the position of the sample pixel and its spectral profile are obtained, and then eq. 8 is used to determine the state of damage.

$$
\forall g_{(x, y)}>0: H_{(x, y)}=C\left(I_{(x, y)}\right) .
$$

Here, $H$ is the classified image, $C$ is the classifier, $I_{(x, y)}$ is the spectral profile, and $g$ is the segmented image.

Next, we proceed to determine the percentage of leaf area with rust (PLAR) quantifying the number of pixels per state of damage; see eq.9.

$$
\forall(H \cap g)_{(x, y)}>0: \operatorname{PLAR}(i)=\sum_{i=1}^{5}(j+1) / N .
$$

Here, PLAR is the percentage of leaf area with rust, $i$ are the levels of rust damage, and $N$ is the number of pixels. 


\subsubsection{Visual analysis}

To conduct the visual analysis (VA), judges belonging to The Phytopathology Laboratory of the Research Institute for the Sustainable Development of Ceja de Selva at National University Toribio Rodríguez de Mendoza of Amazons were used; these judges performed the visual analysis of samples using the SAGARPA scale; see table2.

Table 4. Scale for evaluating rust advance level.

\begin{tabular}{llr}
\hline Level & Description & PLAR \\
\hline 1 & Slightly visible chlorotic spots & $0.5-1$ \\
2 & Visible symptoms of leaf area & $1-5$ \\
3 & The stains begin to bond & $6-20$ \\
4 & The leaves begin to show obvious necrosis & $21-50$ \\
5 & High percentage of necrotic areas & $>50$ \\
\hline Source: extracted from SAGARPA.
\end{tabular}

\subsubsection{Classification method comparison}

In this stage, the results of the classification of validation samples using the trained classifiers and visual classification were compared; it was determined whether there are statistically significant differences between the classification techniques, and the classification method that presents the greatest performance was identified.

\section{Results and Discussion}

\subsection{Preprocessing phase}

In the first steps of the methodology, a total of 22,498 profiles distributed in the pre-established levels were obtained. These profiles were smoothed, the median was extracted, and the upper and lower bounds were determined by adding and subtracting the standard deviation. These profiles are plotted in fig7a.

However, this graph shows excessive overlap between levels that, as commented in Ref. 6, may be due to the manual selection of ROIs, which could have a large impact on the features extracted to describe these regions and logically on the detection accuracy.
Consequently, it was necessary to perform correction by removing the outliers, and the results are plotted in fig $7 \mathrm{~b}$, which shows that the median profiles with or without outliers are the closest, but in the second graph, there is a marked reduction in overlap between levels.

\subsection{Spectral profiles}

The previously obtained median profiles, plotted in fig8, show a constant pattern throughout the development of the infection. In this case, the median profiles, except for the necrotic stage, show intensity values that are progressively higher during the progression of the infection.

In the initial stages of infection, the profiles show a reduction in intensity from 400 to $530 \mathrm{~nm}$ and then an increase in intensity to $670 \mathrm{~nm}$. From this wavelength and up to $1,000 \mathrm{~nm}$, the rate of increase in intensity is slightly lower as the infection progresses. The profile of necrotic tissues, unlike the profiles for the initial, intermediate and advanced stages, starts below the profile for healthy tissues and shows a very low rate of change, similar to that reported by Ref. 19. From the observation of these profiles, it was determined that there is a correspondence between the change in the profiles and the progression of the disease, thereby enabling the analysis of patterns for the later implementation of classifiers.

\subsection{Dimensionality reduction}

Using the Bartlett test, $p$-value $=0$, it was determined that there is a statistically significant difference between the standard deviations of the wavelengths at $99.0 \%$ confidence; likewise, the factor obtained using the Kaiser-Meyer-Olkin test, 0.9813, shows that it is possible and advisable to perform wavelength factorization.

When performing PCA, it was determined that more than $99.1 \%$ of the variance could be explained by two components; see fig9.

Plotting the score of the two selected components, as shown in fig10, it is observed that, except for the profiles with necrotic damage, there is a slight confusion in the limits of each level of damage. 


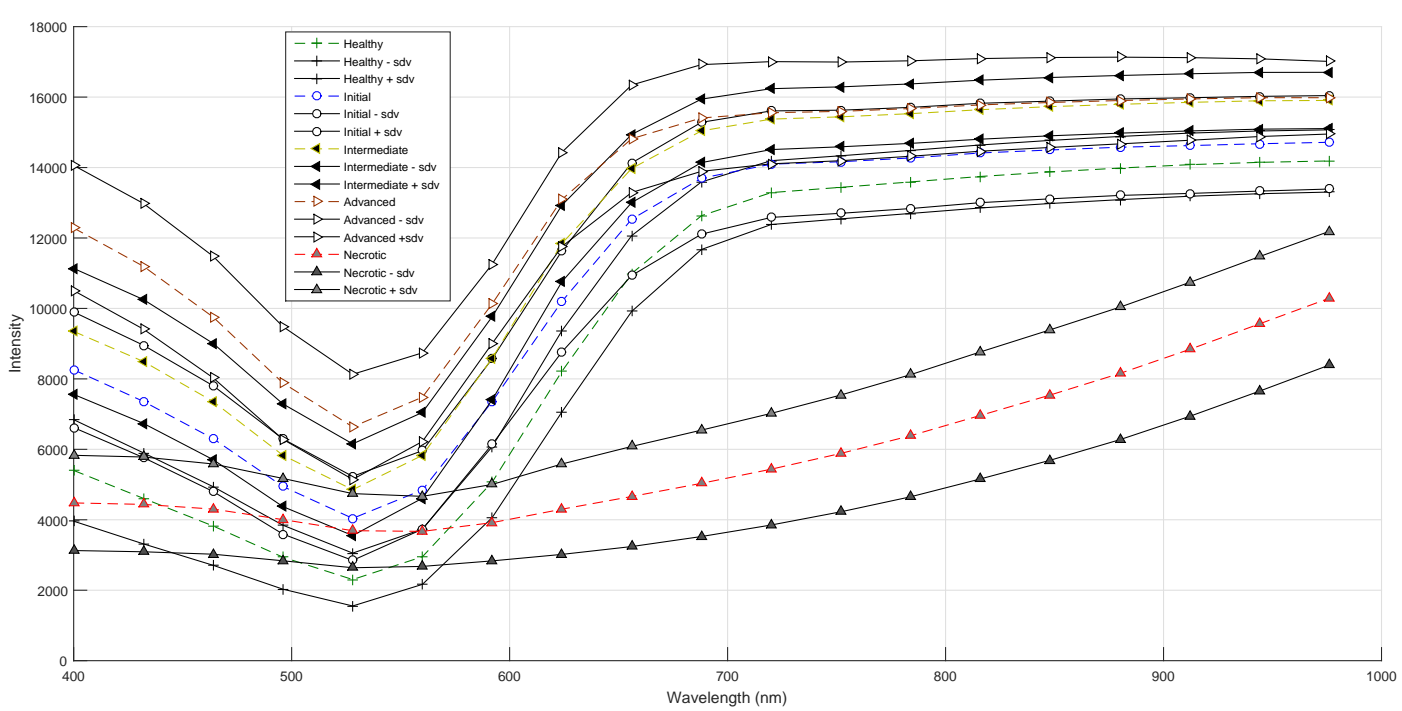

(a)

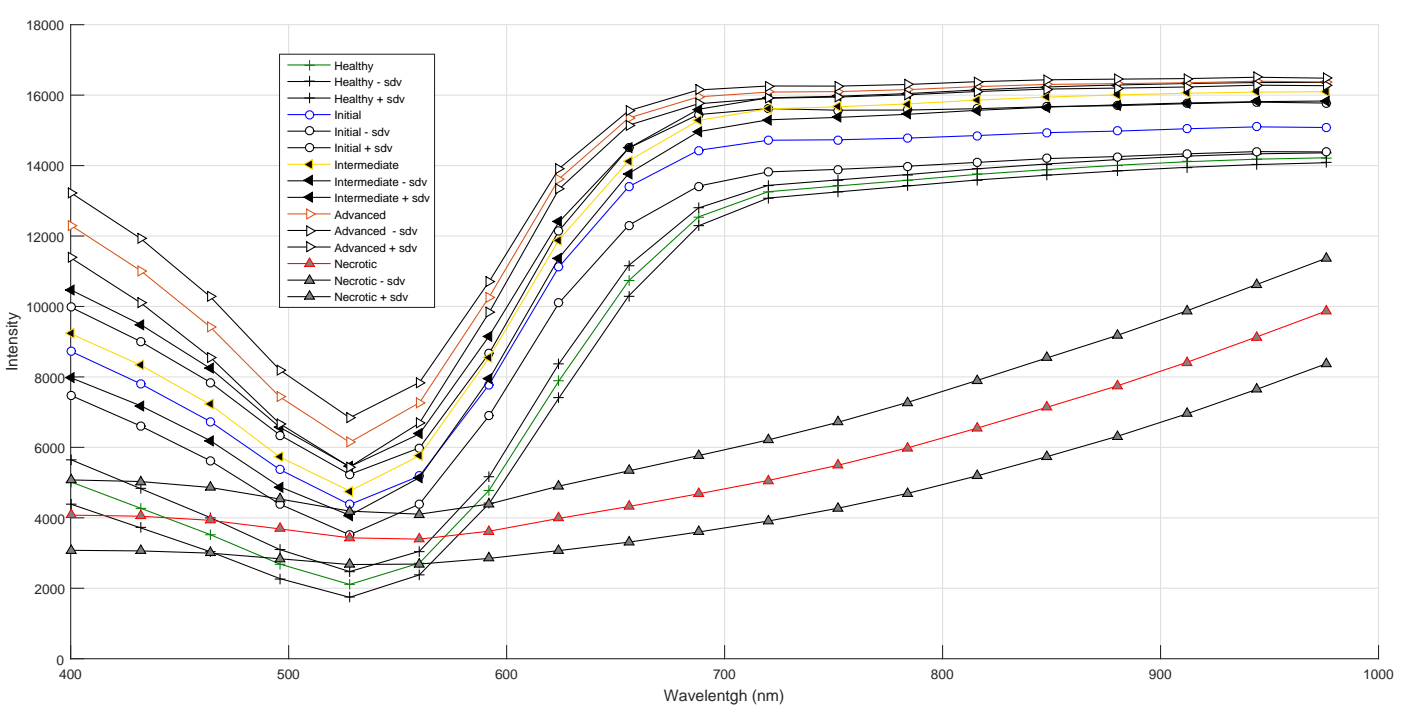

(b)

Fig. 7. Spectral profiles (a) without removal of outliers and (b) with removal of outliers. 


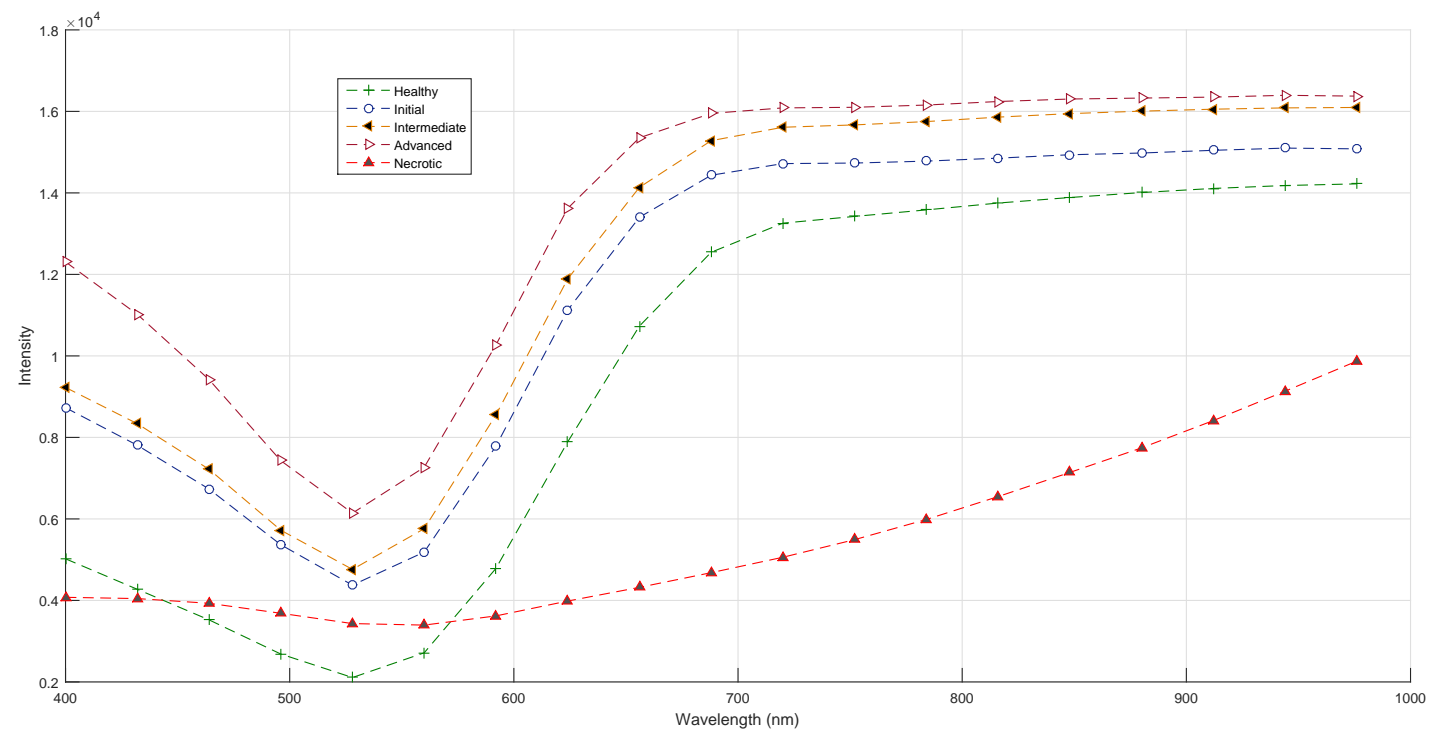

Fig. 8. Average spectral profiles of damage caused by rust.

Table 5. Confusion matrices for classification techniques.

\begin{tabular}{|c|c|c|c|c|c|c|c|}
\hline \multicolumn{8}{|c|}{ Support Vector Machine } \\
\hline & Healthy & Initial & Intermediate & Advanced & Necrotic & \multicolumn{2}{|c|}{ Metrics ${ }^{\star}$} \\
\hline Healthy & $99.20 \%$ & $0.80 \%$ & $0.00 \%$ & $0.00 \%$ & $0.00 \%$ & $A C C:$ & $94.70 \%$ \\
\hline Initial & $2.90 \%$ & $52.40 \%$ & $44.70 \%$ & $0.00 \%$ & $0.00 \%$ & $E R R$ : & $5.30 \%$ \\
\hline Intermediate & $0.00 \%$ & $0.00 \%$ & $\mathbf{9 8 . 5 0} \%$ & $1.50 \%$ & $0.00 \%$ & PRC: & $95.30 \%$ \\
\hline Advanced & $0.00 \%$ & $0.00 \%$ & $3.10 \%$ & $96.90 \%$ & $0.00 \%$ & $F P:$ & $0.60 \%$ \\
\hline Necrotic & $0.00 \%$ & $0.00 \%$ & $0.00 \%$ & $0.00 \%$ & $100.00 \%$ & MAUC: & $96.00 \%$ \\
\hline \multicolumn{8}{|l|}{ Decision Tree } \\
\hline & Healthy & Initial & Intermediate & Advanced & Necrotic & \multicolumn{2}{|c|}{ Metrics $^{\star}$} \\
\hline Healthy & $99.10 \%$ & $0.00 \%$ & $0.00 \%$ & $0.00 \%$ & $0.00 \%$ & ACC: & $90.30 \%$ \\
\hline Initial & $2.60 \%$ & $\mathbf{5 . 5 0} \%$ & $91.80 \%$ & $0.00 \%$ & $0.00 \%$ & ERR: & $9.70 \%$ \\
\hline Intermediate & $0.00 \%$ & $0.00 \%$ & $\mathbf{9 8 . 1 0 \%}$ & $1.90 \%$ & $0.00 \%$ & PRC: & $90.70 \%$ \\
\hline Advanced & $0.00 \%$ & $0.00 \%$ & $2.10 \%$ & $97.90 \%$ & $0.00 \%$ & $F P:$ & $0.50 \%$ \\
\hline Necrotic & $0.00 \%$ & $0.00 \%$ & $0.00 \%$ & $0.00 \%$ & $100.00 \%$ & MAUC: & $86.00 \%$ \\
\hline \multicolumn{8}{|c|}{ K-Nearest Neighbor } \\
\hline & Healthy & Initial & Intermediate & Advanced & Necrotic & \multicolumn{2}{|c|}{ Metrics $^{\star}$} \\
\hline Healthy & $\mathbf{9 8 . 7 0 \%}$ & $1.30 \%$ & $0.00 \%$ & $0.00 \%$ & $0.00 \%$ & $A C C:$ & $93.00 \%$ \\
\hline Initial & $2.80 \%$ & $67.10 \%$ & $30.10 \%$ & $0.00 \%$ & $0.00 \%$ & $E R R$ : & $7.00 \%$ \\
\hline Intermediate & $0.00 \%$ & $10.50 \%$ & $87.20 \%$ & $2.30 \%$ & $0.00 \%$ & PRC: & $96.30 \%$ \\
\hline Advanced & $0.00 \%$ & $0.00 \%$ & $5.00 \%$ & $95.00 \%$ & $0.00 \%$ & $F P:$ & $3.40 \%$ \\
\hline Necrotic & $0.00 \%$ & $0.00 \%$ & $0.00 \%$ & $0.00 \%$ & $100.00 \%$ & MAUC: & 83.00 \\
\hline
\end{tabular}

${ }^{\star}$ ACC: Accuracy; ERR: Error; PRC: Precision; FP: False positive; MAUC: Minimum area under curve. 


\subsection{Classifier creation, training and validation}

The performance of the classification techniques previously mentioned and trained with the two main components determined in the previous step was calculated, and the results are presented in table 3.

The classification techniques used in this paper to differentiate the five stages of rust infection showed precisions between 90 and $94.7 \%$. These results are similar to previously reported results and those shown in table1 for fungal diseases with signs in leaves. In this work, the obtained results were slightly superior to those obtained by Refs. 1 and 48 , who classified three stages of anthracnose infection in strawberry leaves and Fusarium infection in wheat leaves, respectively, but the results were slightly inferior to those reported by Ref. 25 in evaluating tomato leaf infection by Alternaria solani and Helminthosporium oryzae infection over rice leaves. ${ }^{5}$

When analyzing the techniques used in detail through the confusion matrices shown in table3, we observe the following:

- The technique that obtained the highest accuracy (94.7\%) and lowest error (5.3\%) was SVM.

- The decision tree technique obtained the lowest rate of false positives.

- According to the MUAC indicator (an indicator of the performance level of the classifier), the three techniques used showed good performance, with a range between 83 and $96 \%$.

- Referring to the classification between classes, shown in fig11, except for the initial state, the different stages of progression of the infection can be classified using the techniques at an average percentage accuracy of $97.6 \%$.

Based on the results of the $A C C, E R R, F P$ and $M A U C$ metrics, the classifier to be used in the experimentation phase was selected. Consequently, the SVM technique is the one that achieves better benefits for our case study.

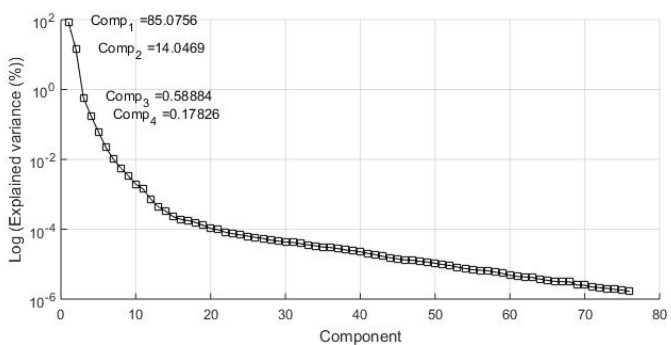

Fig. 9. Variance explained by component.

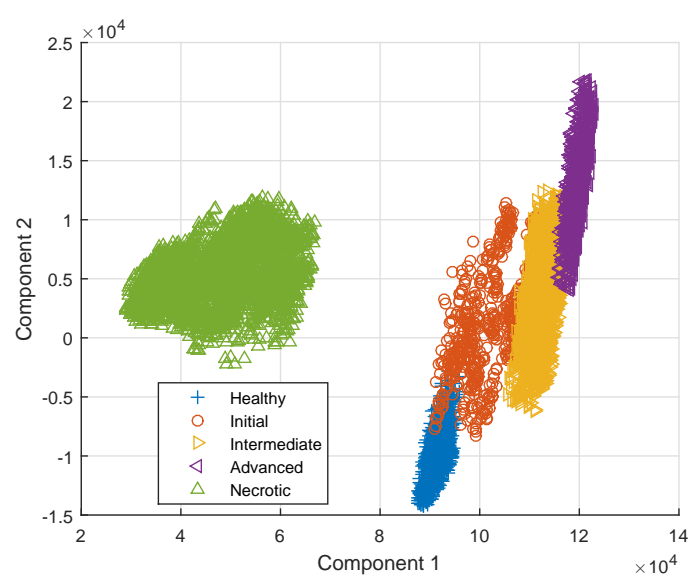

Fig. 10. Levels of infection and components.

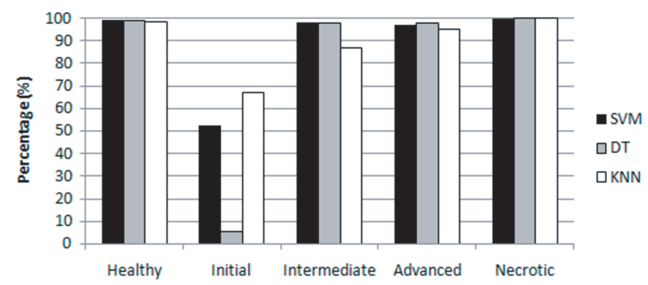

a)

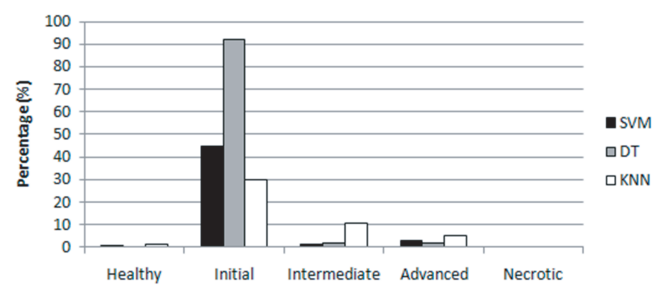

b)

Fig. 11. ACC and $E R R$ when classifying states of damage. a) $A C C$ and b) $E R R$. 


\subsection{Classifier application}

The coffee leaves were analyzed following the guidelines shown in section 2.3.3. For this purpose, the previously trained classifier (SVM) was used, its PLAR was determined, and its respective classified image was generated. fig 12 presents an example of the use of the SVM technique in our case study.

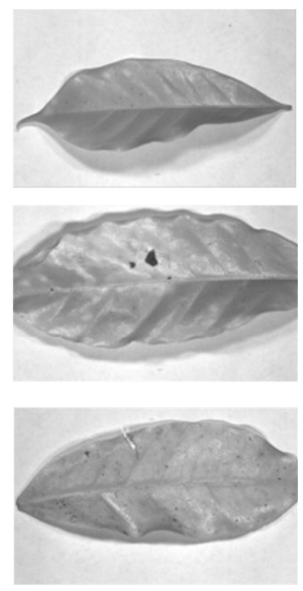

a)
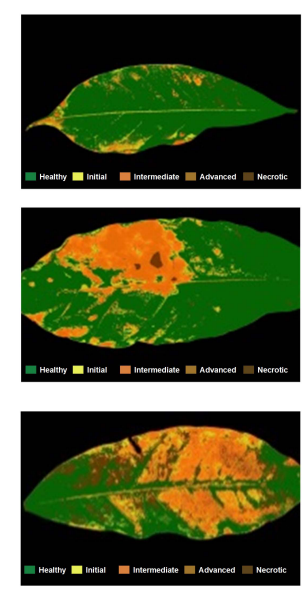

b)
Fig. 12. Classification of rust infection states: a) Intensity images and b) classified images.

From the PLAR value and the limits for leaf damage levels established by SAGARPA, as shown in table2, the classification of the validation samples was performed, obtaining different degrees of infection. fig 13 shows the results for each method used; as shown, there are differences in the results of the applied methods.

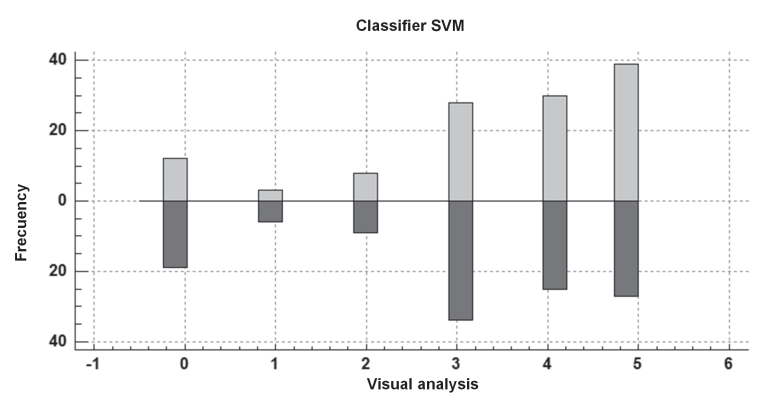

Fig. 13. Frequency of classes obtained by the SVM classifier and visual analysis.

\subsection{Evaluation of classification methods}

Due to differences in the results of the applied methods (SVM and VA), it is necessary to determine whether such differences are significant. For this purpose, a non-parametric statistical analysis was performed using the Kolmogorov-Smirnov test. Consequently, it was determined that there are statistically significant differences. table4 shows the results of the Kolmogorov-Smirnov test in detail.

Subsequently, through the Wilcoxon signed-rank test, the method that generates the highest class values was determined. table 5 presents the results of the Wilcoxon test.

Meanwhile, from the statistics for the VA-SVM ratio, shown in table6, it is observed that there is a statistically significant difference between the VA and the $\operatorname{SVM}(p=0.000<0.05-$ Wilcoxon test $)$. In addition, it was determined that the SVM ratings are significantly higher than those obtained by VA.

Finally, the statistics of both methods were evaluated using samples with a PLAR between 0 and 0.5 and a total of 12 leaves; see table 7.

From these data, it was determined using a Student $t$-test $0.000 \leqslant p<0.05$ at a $95 \%$ confidence level that there is a significant difference between the PLAR obtained with the SVM and the minimum observed with VA. table8 presents the results.

In this way, there is sufficient statistical evidence to confirm that the rigor of evaluation using an expert system based on an SVM classifier is more rigorous than that using VA and the SAGARPA scale. Therefore, it is necessary to develop a new scale for the proposed methodology to increase the effectiveness of rust treatment in coffee plants.

\section{Conclusions}

In this paper, we investigated the potential of using expert systems techniques for classifying different stages of coffee rust infection in hyperspectral images. Five levels of infection were preestablished (healthy, initial, intermediate, advanced 
Table 6. Kolmogorov-Smirnov test for classification.

\begin{tabular}{llll}
\hline \multicolumn{2}{c}{ Statistical } & Classifier SVM & Visual Analysis \\
\hline$N$ & & 120 & 120 \\
\hline Normal parameters $^{(a)}$ & Media & 3.49 & 3.01 \\
& Standard deviation & 1.55 & 1.68 \\
& Absolute & 0.21 & 0.22 \\
\hline More extreme differences ${ }^{(b)}$ & Positive & 0.17 & 0.12 \\
& Negative & -0.21 & -0.22 \\
\hline The Kolmogorov-Smirnov $Z$ & & 2.32 & 2.35 \\
\hline Asymp.sig. (2-tailed) & & 0 & 0 \\
\hline${ }^{(a)}$ The contrast distribution is normal. ${ }^{(b)}$ Data from both variables are normal $(0.00 \geqslant p<0.05)$.
\end{tabular}

Table 7. Wilcoxon signed-rank test.

\begin{tabular}{lllr}
\hline Visual - SVM & $N$ & Average rank & Sum of ranks \\
\hline Negative rank & $52^{(a)}$ & 27.07 & 1407.50 \\
Positive rank & $1^{(b)}$ & 23.5 & 23.5 \\
Draws & $67^{(c)}$ & & \\
\hline Total & 120 & \\
\hline${ }^{(a)}$ Visual $<$ SVM. ${ }^{(b)}$ Visual $>$ SVM. ${ }^{(c)}$ Visual $=$ SVM.
\end{tabular}

Table 8. The contrast statistics for the VA-SVM ratio.

\begin{tabular}{lr}
\hline Statistical & Visual - SVM \\
\hline$Z$ & $-6.687^{(a)}$ \\
Asymptotic Sig. (bilateral) & $0.000^{(b)}$ \\
\hline${ }^{(a)}$ Based on positive ranges. ${ }^{(b)}$ Based on the Wilcoxon signed-rank test.
\end{tabular}

Table 9. Statistics of the sample PLAR.

\begin{tabular}{llll}
\hline$N$ & Median & Standard deviation & Standard Error \\
\hline 12 & 0.1167 & 0.103 & 0.030 \\
\hline
\end{tabular}

Table 10. T-test for a sample.

\begin{tabular}{lccccr}
\hline$t$ & $g l$ & Sig. (bilateral) & Difference of means & \multicolumn{2}{c}{$\begin{array}{l}\text { Confidence interval for the difference (95\%) } \\
\text { lower }\end{array}$} \\
\hline-12.894 & 11 & 0.000 & -0.38333 & -0.4488 & -0.3179 \\
\hline
\end{tabular}


and necrotic), which were used to create and train three classifiers (DT, SVM, and KNN). The results of the experiment revealed differences in their mean spectral profiles. The classifier based on the SVM technique obtained the best classification performance $(A C C=94.7 \%$, and $F P=0.6 \%)$. The results obtained using the SVM classifier were statistically compared against the results obtained through visual analysis, and a statistically significant difference was observed. The results have confirmed the feasibility of using expert systems techniques and hyperspectral images for evaluating the progression of coffee rust infection.

\section{Acknowledgments}

This work has been funded by the Fund for Innovation, Science, and Technology (FINCyT) through the Project Contract 220-IA-2013. The fourth author thanks CONACYT-Mexico for the support to participate in this research work through project 2143Fortalecimiento de las capacidades de TICs en $\mathrm{Na}$ yarit.

\section{References}

1. Y. Yeh, W. Chung, J. Liao, C. Chung, Y. Kuo, and T. Lin. Strawberry foliar anthracnose assessment by hyperspectral imaging. Comput Electron Agric, 122:1 $-9,2016$.

2. S. Sankaran, A. Mishra, R. Ehsani, and C.stina Davis. A review of advanced techniques for detecting plant diseases. Comput Electron Agric, 72(1):1-13, 2010.

3. D. Al-Bashish, M. Braik, and S. Bani-Ahmad. A framework for detection and classification of plant leaf and stem diseases. In Signal and Image Processing (ICSIP), 2010 International Conference on, pages 113-118. IEEE, 2010.

4. M. Ray, A. Ray, S. Dash, A. Mishra, K. G. Achary, S. Nayak, and S. Singh. Fungal disease detection in plants: Traditional assays, novel diagnostic techniques and biosensors. Biosens Bioelectron, 87:708$723,2017$.

5. Z. Liu, J. Huang, and R. Tao. Characterizing and estimating fungal disease severity of rice brown spot with hyperspectral reflectance data. Rice Science, 15(3):232-242, 2008.

6. J. G. A. Barbedo, L. V. Koenigkan, and T. T. Santos. Identifying multiple plant diseases using digital image processing. Biosyst Eng, 147:104-116, 2016.
7. T. Rumpf, A. Mahlein, U. Steiner, E. Oerke, H. Dehne, and L. Plümer. Early detection and classification of plant diseases with support vector machines based on hyperspectral reflectance. Comput Electron Agric, 74(1):91-99, 2010.

8. S. Serranti, D. Cesare, and G. Bonifazi. The development of a hyperspectral imaging method for the detection of Fusarium-damaged, yellow berry and vitreous italian durum wheat kernels. Biosyst Eng, 115(1):2030, 2013.

9. D. L. Borges, S. T. C. de M. Guedes, A. R. Nascimento, and P. Melo-Pinto. Detecting and grading severity of bacterial spot caused by Xanthomonas spp. in tomato (Solanum lycopersicon) fields using visible spectrum images. Comput Electron Agric, 125:149$159,2016$.

10. E. B. Knipling. Physical and physiological basis for the reflectance of visible and near-infrared radiation from vegetation. Remote Sens Environ, 1(3):155-159, 1970.

11. D. Moshou, C. Bravo, R. Oberti, J. S. West, H. Ramon, S. Vougioukas, and D. Bochtis. Intelligent multisensor system for the detection and treatment of fungal diseases in arable crops. Biosyst Eng, 108(4):311321, 2011.

12. G. M. ElMasry and S. Nakauchi. Image analysis operations applied to hyperspectral images for noninvasive sensing of food quality-a comprehensive review. Biosyst Eng, 142:53-82, 2016.

13. J. G. A. Barbedo. A review on the main challenges in automatic plant disease identification based on visible range images. Biosyst Eng, 144:52-60, 2016.

14. H. R. Xu, Y. B. Ying, X. P. Fu, and S. P. Zhu. Nearinfrared spectroscopy in detecting leaf miner damage on tomato leaf. Biosyst Eng, 96(4):447-454, 2007.

15. D. Wu, L. Feng, C. Zhang, and Y. He. Early detection of botrytis cinerea on eggplant leaves based on visible and near-infrared spectroscopy. Trans ASABE, 51(3):1133-1139, 2008.

16. J. Blasco, N. Aleixos, J. Gómez-Sanchis, and E. Moltó. Recognition and classification of external skin damage in citrus fruits using multispectral data and morphological features. Biosyst Eng, 103(2):137145, 2009.

17. P. Williams, P.1 Geladi, G. Fox, and M. Manley. Maize kernel hardness classification by near infrared (NIR) hyperspectral imaging and multivariate data analysis. Anal Chim Acta, 653(2):121-130, 2009.

18. Z. Liu, H. Wu, and J. Huang. Application of neural networks to discriminate fungal infection levels in rice panicles using hyperspectral reflectance and principal components analysis. Comput Electron Agric, 72(2):99-106, 2010.

19. C. Yang, J. H. Everitt, and C. J. Fernandez. Comparison of airborne multispectral and hyperspectral 
imagery for mapping cotton root rot. Biosyst Eng, 107(2):131-139, 2010.

20. E. Bauriegel, H. Brabandt, U. Gärber, and W. B. Herppich. Chlorophyll fluorescence imaging to facilitate breeding of bremia lactucae-resistant lettuce cultivars. Comput Electron Agric, 105:74 - 82, 2014.

21. J. Zhang, R. Pu, J. Wang, W. Huang, L. Yuan, and J. Luo. Detecting powdery mildew of winter wheat using leaf level hyperspectral measurements. Comput Electron Agric, 85:13 - 23, 2012.

22. J. Zhang, L. yuan, R. Pu, R. W. Loraamm, G. Yang, and J. Wang. Comparison between wavelet spectral features and conventional spectral features in detecting yellow rust for winter wheat. Comput Electron Agric, 100:79 - 87, 2014.

23. R. Zhou, S. Kaneko, F. Tanaka, M. Kayamori, and M. Shimizu. Disease detection of cercospora leaf spot in sugar beet by robust template matching. Comput Electron Agric, 108:58-70, 2014.

24. B. Jaillais, P. Roumet, L. Pinson-Gadais, and D. Bertrand. Detection of Fusarium head blight contamination in wheat kernels by multivariate imaging. Food Control, 54:250-258, 2015.

25. C. Xie, Y. Shao, X. Li, and Y. He. Detection of early blight and late blight diseases on tomato leaves using hyperspectral imaging. Sci Rep, 5:1-11, 2015.

26. D. Wu and D. Sun. Advanced applications of hyperspectral imaging technology for food quality and safety analysis and assessment: A review, Part I: Fundamentals. Innov Food Sci Emerg Technol, 19:15 28, 2013.

27. J. Avelino, H. Zelaya, A. Merlo, A. Pineda, M. Ordoñez, and S. Savary. The intensity of a coffee rust epidemic is dependent on production situations. Ecol Model, 197(34):431 - 447, 2006.

28. D. Jackson, J. Skillman, and J. Vandermeer. Indirect biological control of the coffee leaf rust, Hemileia vastatrix, by the entomogenous fungus Lecanicillium lecanii in a complex coffee agroecosystem. Biol Control, 61(1):89 - 97, 2012.

29. B. Heredia. Recomendaciones para el combate de la roya del café (Hemileia vastatrix Berk et $B r$.). San José - Costa Rica. Instituto del café de Costa Rica, Centro de Investigaciones en Café (CICAFE), 3ra edition, 2013.

30. J. Brown, J. H. Whan, M. K. Kenny, and P. R. Merriman. The effect of coffee leaf rust on foliation and yield of coffee in papua new guinea. Crop Prot, 14(7):589 - 592, 1995.

31. F. Haddad, L. A. Maffia, E. S. G. Mizubuti, and H. Teixeira. Biological control of coffee rust by antagonistic bacteria under field conditions in brazil. Biol Control, 49(2):114 - 119, 2009.

32. G. Polder, G. W. A. M. van der Heijden, J. van Doorn, and T. A. H. M. C. Baltissen. Automatic detection of tulip breaking virus (TBV) in tulip fields using machine vision. Biosyst Eng, 117:35 - 42, 2014.

33. G. Polder, G. W. A. M. van der Heijden, J. van Doorn, J. G. P. W. Clevers, R. van der Schoor, and A. H. M. C. Baltissen. Detection of the tulip breaking virus (TBV) in tulips using optical sensors. Precis Agric, 11(4):397-412, 2010.

34. W. Lee, M. S. Kim, H. Lee, S. R. Delwiche, H. Bae, D. Kim, and B. Cho. Hyperspectral near-infrared imaging for the detection of physical damages of pear. J Food Eng, 130:1 - 7, 2014.

35. S. Fukuda, E. Yasunaga, M. Nagle, K. Yuge, V. Sardsud, W. Spreer, and J. Müller. Modelling the relationship between peel colour and the quality of fresh mango fruit using random forests. J Food Eng, 131:7 $-17,2014$.

36. L. Ravikanth, C. B. Singh, D. S. Jayas, and N. D. G. White. Classification of contaminants from wheat using near-infrared hyperspectral imaging. Biosyst Eng, 135:73 - 86, 2015.

37. W. Jun, M. S. Kim, K. Lee, P. Millner, and K. Chao. Assessment of bacterial biofilm on stainless steel by hyperspectral fluorescence imaging. Sens \& Instrumen Food Qual, 1(3):41 - 48, 2009.

38. G. Naganathan, K. Cluff, A. Samal, C. Calkins, D. Jones, C. Lorenzen, and J. Subbiah. Hyperspectral imaging of ribeye muscle on hanging beef carcasses for tenderness assessmen. Comput Electron Agric, 116:55 - 64, 2015.

39. T. Qiao, J. Ren, C. Craigie, J. Zabalza, C. Maltin, and S. Marshall. Singular spectrum analysis for improving hyperspectral imaging based beef eating quality evaluation. Comput Electron Agric, 115:21-25, 2015.

40. J. G. A. Barbedo, C. S. Tibola, and J. M. C. Fernandes. Detecting Fusarium head blight in wheat kernels using hyperspectral imaging. J Food Eng, 131:65 76, 2015.

41. A. Fernandes, J. Lousada, J. Morais, J. Xavier, J. Pereira, and P. Melo-Pinto. Chemical-free assessment and mapping of major constituents in beef using hyperspectral imaging. J. Food Eng., 117:235 - 246, 2013.

42. D. Liu, D. Sun, and X. Zeng. Recent advances in wavelength selection techniques for hyperspectral image processing in the food industry. Food Bioprocess Technol, 7(2):307-323, 2014.

43. G. ElMasary and P. Sun, D. Allen. Chemical-free assessment and mapping of major constituents in beef using hyperspectral imaging. J Food Eng, 117:235 246, 2013.

44. T. Kimiya, A. H. Sivertsen, and K. Heia. VIS/NIR spectroscopy for non-destructive freshness assessment of atlantic salmon (Salmo salar L.) fillets. J Food Eng, 116(3):758 - 764, 2013.

45. F. J. Rodríguez-Pulido, B. Gordillo, M. L. González- 
Miret, and F. J. Heredia. Analysis of food appearance properties by computer vision applying ellipsoids to colour data. Comput Electron Agric, 99:108 - 115, 2013.

46. W. Wang, X. Ni, K. C. Lawrence, s. Yoon, G. W. Heitschmidt, and P. Feldner. Feasibility of detecting aflatoxin B1 in single maize kernels using hyperspectral imaging. J Food Eng, 166:182 - 192, 2015.
47. SAGARPA. Ficha técnica de la roya del cafeto Hemileia vastatrix Berkeley \& Broome. Online, 2013. México: Secretaría General de Agricultura Desarrollo Rural Pesca y Alimentación.

48. E. Bauriegel, A. Giebel, M. Geyer, U. Schmidt, and W. B. Herppich. Early detection of Fusarium infection in wheat using hyper-spectral imaging. Comput Electron Agric, 75(2):304-312, 2011. 